\title{
LHyCon (Low Hydrogen Concentration measurement sensor)
}

\author{
Arvind Balakrishnan ${ }^{1}$, Saner Akan ${ }^{1}$, Marko Völkel1, Sebastian Geiger², Peter Rabenecker2, \\ ${ }^{1}$ LAMTEC Meß- und Regeltechnik für Feuerungen GmbH \& Co. KG, Wiesenstraße 6, Germany \\ ${ }^{2}$ Fraunhofer-Institut für Chemische Technologie ICT, Joseph-von-Fraunhofer-Str. 7, Germany
}

\begin{abstract}
This paper presents a newly developed Low Hydrogen Concentration measurement sensor for hydrogen leak detection. This project further developed a solid electrolyte sensor based on mixed potential theory. The main goals of this project were to develop a hydrogen sensor that has an increased sensitivity for $0-1000 \mathrm{ppb}$ of Hydrogen in air, nearly linear sensor signal for 0-1000 ppb Hydrogen in air, quick response $\left(\mathrm{t}_{60}<1 \mathrm{sec}\right.$ ), low-cost production and reduced power consumption and portability through miniaturization. The senor type LH 44 which was fabricated within the framework of this project was found to be the most efficient variation which fulfils the defined goals. The final goal of the project, to develop a low concentration hydrogen sensor for mobile application through miniaturization, was demonstrated.
\end{abstract}

Keywords: Low Hydrogen Concentration measurement sensor, solid-state mixed potential electrochemical sensors, miniaturized hydrogen sensor and mobile application

\section{Introduction}

Components tightness testing is one of the industrial applications where helium is most commonly used as tracer gas [1]. But its drawbacks are high cost and requiring vacuum environment as well as a sophisticated measurement set up. Hydrogen is the lightest element followed by helium. Further, it is cheaper compared to Helium and can be detected easily in ambient air. Hence, its presence has increased in the last decades in this field of application. This has also increased the demand for a hydrogen sensor that is sensitive for low hydrogen concentration with a quick response time and a possible low cost production. Solid-state mixed potential hydrogen sensors became increasingly popular in the last three decades because they offer a simple means to measure hydrogen with high sensitivity and quick response time [2] [3]. A typical sensor of this sort consists of two different electrodes with a solid-state electrolyte in between [4]. The working principle, known as non-Nernst-behaviour [3], is based on a voltage generated due to the different electrochemical behaviour of the two electrodes [2].

The CarboSen sensor of LAMTEC GmbH is one of such solid-state mixed potential electrochemical sensors that has a wide field of application [5]. Depending on the application CarboSen is also referred as HydroSen [6]. The cross-section schematic view of CarboSen sensor is shown in Figure 1. A stacked assembly of working electrode - solid-state electrolyte - reference electrode is realized on an aluminium oxide $\left(\mathrm{Al}_{2} \mathrm{O}_{3}\right)$ carrier substrate. Reference electrode is a platinum and the working electrode is a platinum-gold based cermet. The solid-state electrolyte used here is yttria stabilized zirconia (YSZ) and has a porous structure. The porous structure enables both electrodes to react with the measured gas simultaneously. YSZ which has to be operated in a temperature range between $400{ }^{\circ} \mathrm{C}$ and $900{ }^{\circ} \mathrm{C}$ in order to utilize oxygen ion conductivity [3]. Therefore, a heater is placed on the back side of the carrier substrate.

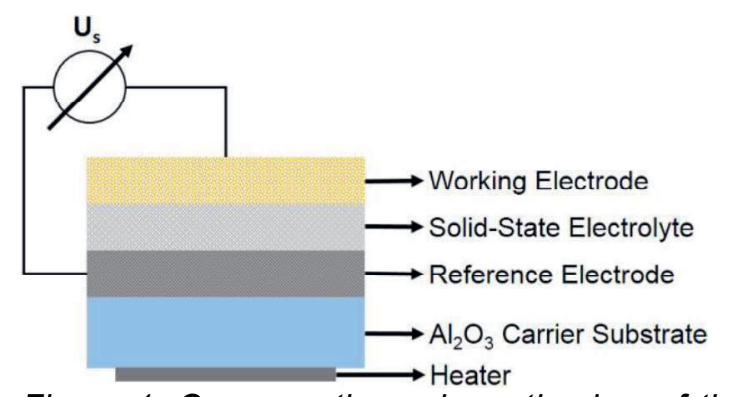

Figure 1: Cross-section schematic view of the CarboSen sensor construction

The working electrode and the reference electrode react to hydrogen in different ways. On the reference electrode, only catalytic reactions occur but on the working electrodes, both catalytic and electrochemical reactions 
occur. This builds a measureable potential difference between the electrodes, which yields the sensor signal. As the hydrogen concentration changes, the mixed potentials of each electrode also change due to the change in the rate of the catalytic and electrochemical reactions.

One of the limitations of CarboSen is the unsatisfactory sensitivity for low Hydrogen concentrations at ambient air. A satisfactory measured sensor voltage is delivered by CarboSen only from the range of $10-1000$ ppm of Hydrogen in ambient air.

Basing this as the motivation the focus of the LHyCon project was to develop a sensor system which reaches the following goals

- Increased sensitivity for 0-1000 ppb of Hydrogen in air.

- Nearly linear sensor signal for 0-1000 ppb Hydrogen in air (optional).

- Response time ( $\left.\mathrm{t}_{60}<1 \mathrm{sec}\right)$.

- Low-cost production.

- Reduced power consumption and portability through miniaturization.

\section{Approach}

The variation of surface morphology and the optimization of the functional materials corresponding to the electrode 1 and 2 were identified to be a suitable approach to reach the above-mentioned goals.

A design of experiments was used to generate a test matrix with the below-mentioned aspects as main focus. The test matrix was classified into 6 major sections, each section corresponds to one of the below-mentioned aspects.

- Variation of the functional electrode material thickness of electrode 2 .

- Variation of the available three phase boundary on the functional electrode 2 .

- Variation of the different functional material-I, on electrode 2.

- Variation of the different functional material-II, on electrode 2 .

- Variation of the surface morphology of electrode 2 by fabrication process.

- Variation the electrode design of electrode 1.

Within each of the sections, there were certain variations. Details of each of these sensor variations will not be revealed in this paper due to the intellectual property rights of LAMTEC $\mathrm{GmbH}$.

The nomenclature used for each test section was LH XX. LH stands for LHyCon, the name of the project and the $X X$ corresponds to the test number. In total 75 different sensor types were tested on the available state-of-the-art CarboSen sensor platform.

\section{Sensor fabrication}

The sensors, fabricated in this project, are a planar type solid-state mixed potential electrochemical sensors. Planar sensors were fabricated with screen printing technology and industrially available $\mathrm{Al}_{2} \mathrm{O}_{3}$ was chosen as the carrier substrate by maintaining flexibility, reproducibility and the possibility for low-cost mass production. On one side of the carrier substrate two electrodes with contact pads and glass protective coating, on the other side the heater element with contact pads and glass protective coating were screen printed.

In the state-of-the-art CarboSen sensor, the solid-state electrolyte is YSZ, reference electrode is fabricated using an industrially available high purity platinum paste and the working electrode is a platinum-gold alloy cermet material and is fabricated using a hybrid fabrication process. The heating element is also screen printed using a platinum paste. Each layer of screen printing was followed by a high temperature sintering process. The Schematic view of the state-of-the-art CarboSen is shown in Figure 2.

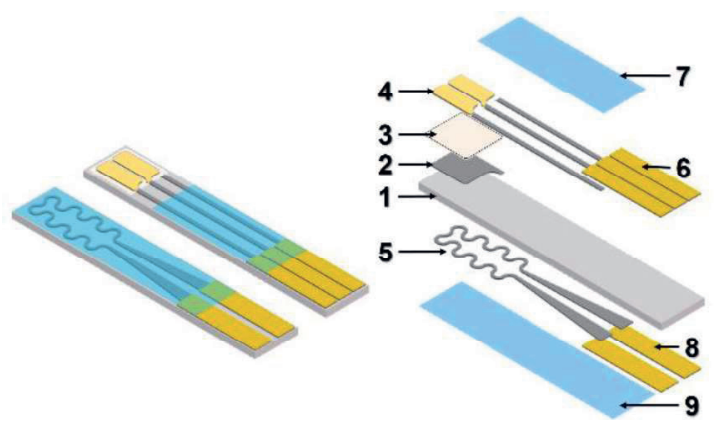

Figure 2: Schematic view of the state-of-the-art CarboSen sensor design.

1: carrier substrate, 2: reference electrode, 3: YSZ, 4: Working electrodes, 5: heating element, 6: electrode contact pads, 7: glass layer, 8: heating element contact pads and 9: glass layer.

The protective glass layer is used on the front side to protect the contact lines that extend from the electrodes to the gold contact pads. Similarly, on the back side the complete surface of the heating element is covered with the protective glass layer. The sensors fabricated in the LHyCon project were based on the same sensor design and construction as in the CarboSen, but the electrodes were varied according to the test matrix. One sensor type variation from the test matrix was fabricated on 
one a 4" * 4" carrier substrate which consisted of 75 sensor elements.

\section{Characterisation}

A picture of a fully fabricated LHyCon sensor is presented in Figure 3.

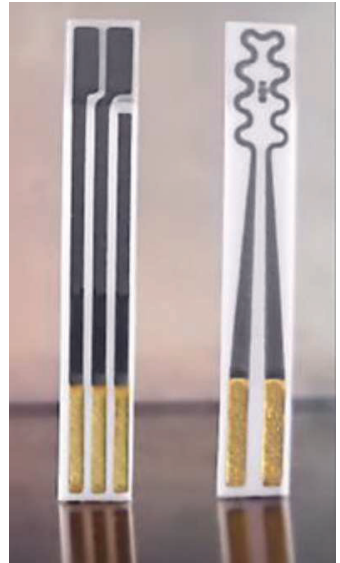

Figure 3: Fully fabricated LHyCon sensor.

In Figure 3, the left-hand side image is the front side of the sensor and the right-hand side image is the backside. Figure 4 shows the cross-section SEM image of the fabricated sensor element

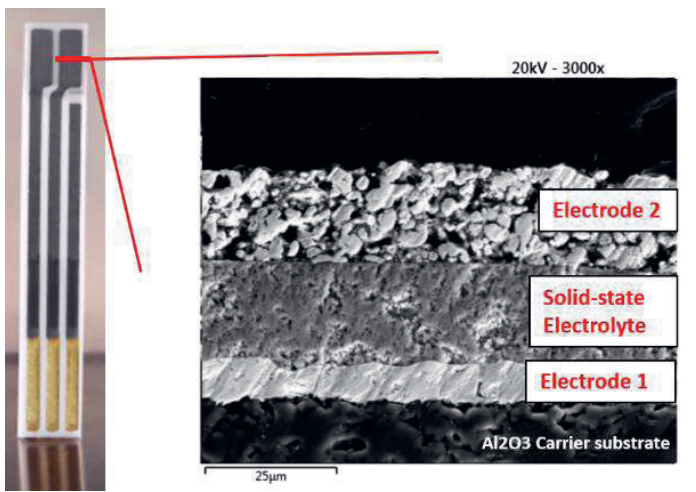

Figure 4: Cross-section SEM image of a specific location on the fabricated sensor.

\section{Performance}

The sensor elements were tested with good statistics (10 sensor elements simultaneously from every type) in various low concentrations of hydrogen in air and different sensor operating temperatures by yielding temperature characteristics as well as test gas characteristics of the fabricated sensor elements. Connected to a 10-fold sensor electronic rack, in a 10-fold measurement chamber the sensor elements were exposed to test gas concentrations that were well-defined through commissioning premixed test gas cylinders and high precision pc controlled digital flow regulators. A schematic view of the measurement setup is presented in Figure 5.

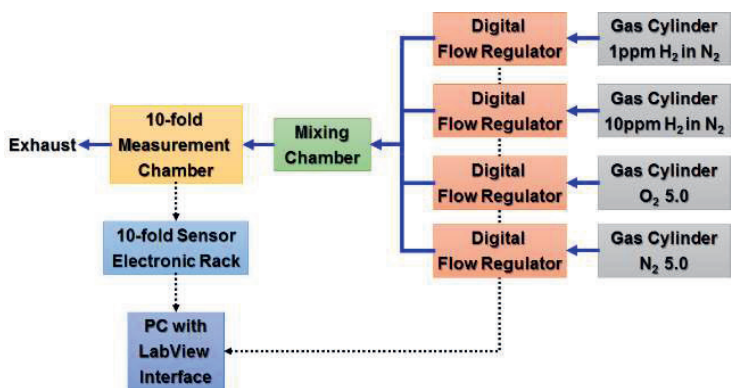

Figure 5: Test gas measurement setup

The 10-fold measurement chamber was an assembly, which consisted of two rectangular PEEK plates pressed on each other by a pneumatic actuator forming 10 individual slots as measurement chambers for each sensor element.

One of the rectangular PEEK plates was stationary on one end of the pneumatic actuator with sensor sockets and cables attached. The other one was the moving counter plate. The pneumatic actuator was operated on one horizontal axis through a control unit with an input of 4 bar air pressure. A picture of the 10 -fold measurement chamber is presented in Figure 6.

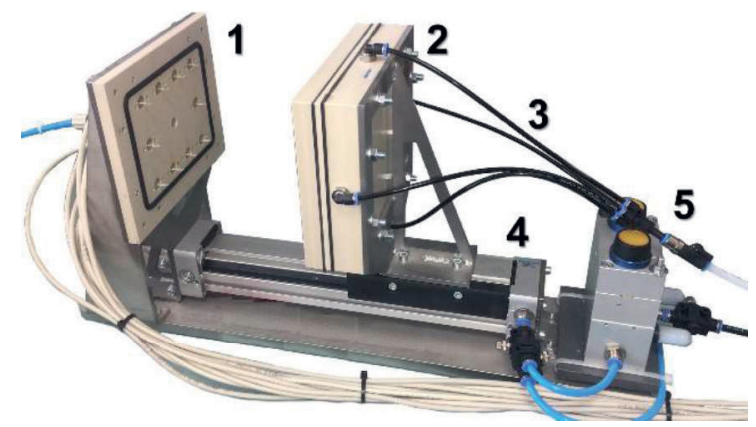

Figure 6: 10-fold measurement chamber,

1: plate with sensor connection, 2 : counter plate with gas divider, 3: test gas connections, 4: Pneumatic actuator and 5: Pneumatic control with pressurized air.

All tests were carried out with three concentration ranges:

- 0-1000 ppb of hydrogen in air

- 1-7 ppm of hydrogen in air

- $7-100$ ppm of hydrogen in air

The test gas cylinders with low concentrations of hydrogen and the gas mixing assembly were cross-verified using a mass-spectrometer. The mass spectrometer was able to detect concentrations lower than $1 \mathrm{ppm}$ hydrogen in synthetic air (down to $50 \mathrm{ppb}$ ) that were mixed and supplied by the LHyCon gas mixing setup. Through a PC with LabView software interface, at each measurement point test gas mass flow 
rate was fixed at $1600 \mathrm{sccm}$ as well as the dwell time at 3 minutes.

The sensors were connected to the 10-fold sensor electronic Figure 7 where the sensor temperatures were manually adjusted and measurement data was recorded through a PC with LabView interface.

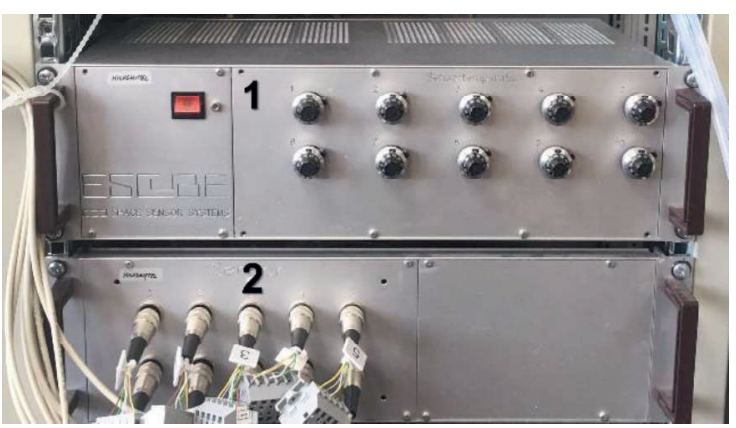

Figure 7: 10-fold Sensor Electronic Rack.

1: potentiometers for adjusting sensor temperature and 2: sensor connection module

Due to the nature of solid-state electrolytes, in the same hydrogen gas concentration the sensors perform differently at different operating temperatures. Therefore, temperature characteristics were obtained through various tests. The sensor temperature was controlled by adjusting the heater power and further control the heater resistance at a constant value. For the tests, three main heater powers values were specified: $2,1 \mathrm{~W}, 2,5 \mathrm{~W}$ and $2,9 \mathrm{~W}$ corresponding approximately to $450^{\circ} \mathrm{C}, 550^{\circ} \mathrm{C}$ and $600^{\circ} \mathrm{C}$ respectively. The optimum heater power for low concentrations of hydrogen in air was found at $2,1 \mathrm{~W}$. The following measurement procedure was followed uniformly for all the sensors measured in the project:

- $\quad$ Pre-heating with $3 \mathrm{~W}$ heating power for a defined time to initialize the sensor.

- Sensor heating set to $2,9 \mathrm{~W}$ and the sensor signal measurement when exposed to $0-100 \mathrm{ppm}$ of hydrogen concentration in air.

- Sensor signal measurement when exposed to 0-7 ppm of hydrogen concentration in air with heating powers $2,9 \mathrm{~W}, 2,5 \mathrm{~W}$ and $2,1 \mathrm{~W}$.

- Sensor heating set to $2,1 \mathrm{~W}$ and the sensor signal measurement when exposed to 1-1000 ppb of hydrogen concentration in air.

\section{Results and discussion}

All fabricated sensors were measured using the three sets of different hydrogen concentration ranges in air. The measured results were analysed and concluded that the sensor type LH 44 best fulfilled the defined goals of the
LHyCon project. The average measured sensor voltage of $10 \mathrm{LH} 44$ sensors when subjected to hydrogen concentrations of $0 \mathrm{ppm}$ to $7 \mathrm{ppm}$ is presented in Figure 8, where the heating power is $2,1 \mathrm{~W}$.

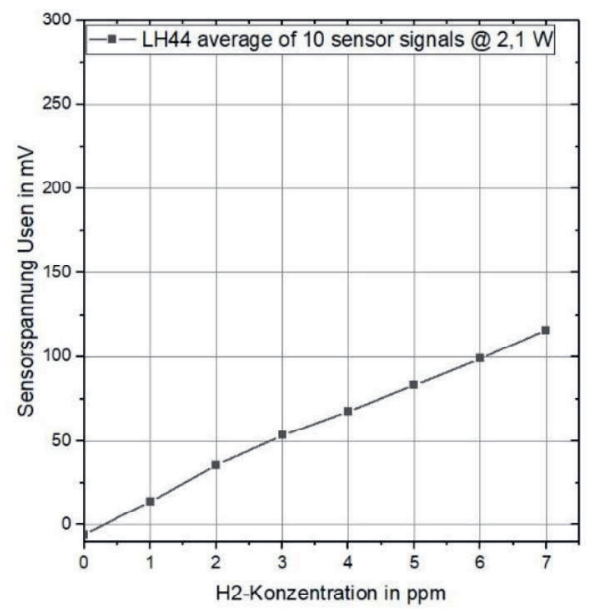

Figure 8: Sensor signal characteristic of LH 44 sensor type when exposed with 0-7 ppm hydrogen concentrations in air

Consecutively, the average measured sensor signal of 10 sensors of LH 44 sensor type when subjected to hydrogen concentration of $0 \mathrm{ppb}$ to $1000 \mathrm{ppb}$ is presented in Figure 9. The figure represents the behaviour of the sensor signal when the sensors were operated with heating power of $2,1 \mathrm{~W}$.

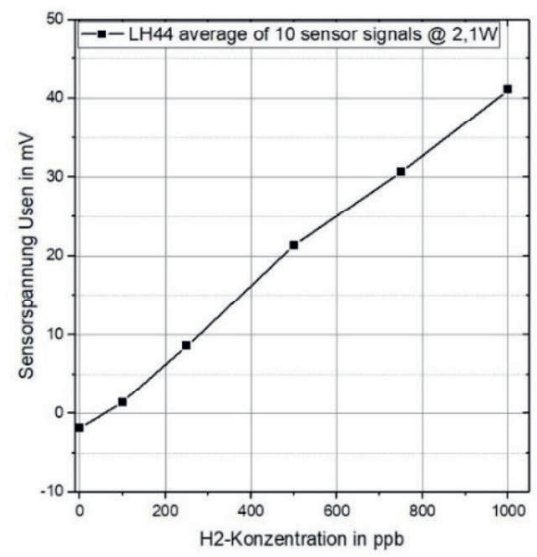

Figure 9: Sensor signal characteristic of LH 44 sensor type when exposed with 0-1000 ppb hydrogen concentrations in air

In order to the compare the amplitude of the average measured sensor signals of the LH 44 sensor type in $0 \mathrm{ppb}$ to $1000 \mathrm{ppb}$ range, 10 state-of-the-art CarboSen sensors were tested exactly as mentioned previously. The comparison of the average measured sensor 
signals is presented in Figure 10. This figure reveals the increase in the sensor signal for the LH 44 sensor type in comparison to the CarboSen. This increase in the sensor signal is attributed to an increased electrochemical reactions dominated and reduced catalytic reactions of the LH 44 sensor type electrode surface.

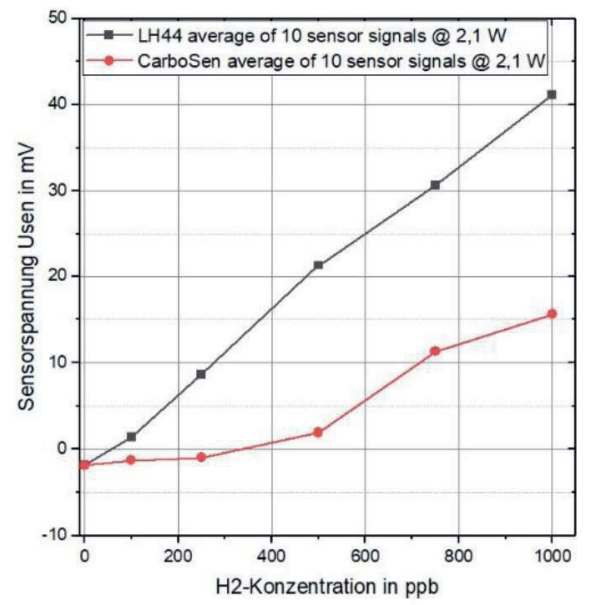

Figure 10: Comparison of sensor signals of LH 44 sensor type and the CarboSen when exposed to the hydrogen concentration range 0-1000 ppb.

Based on this inference, the final goal of the project was carried out, to achieve a low concentration hydrogen sensor for mobile application through miniaturization of the sensor that has reduced power consumption and better portability.

The miniaturized version of the LH44 sensor was designed with $3 \mathrm{~mm} * 3 \mathrm{~mm}$ substrate dimensions. Therefore, the new sensor type is named as LH 44X3. The exploded schematic view of the miniaturized LH 44X3 sensors is shown in Figure 11.

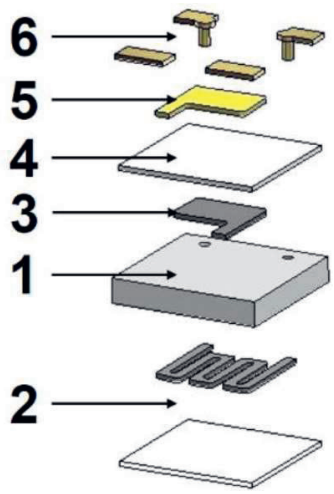

Figure 11: Exploded schematic representation of miniaturised LH 44X3 sensors.
1: carrier substrate, 2: heating element, 3: electrode 1, 4: solid-state electrolyte YSZ, 5: electrode 2 and 6 : gold contact pads and the via hole connections.

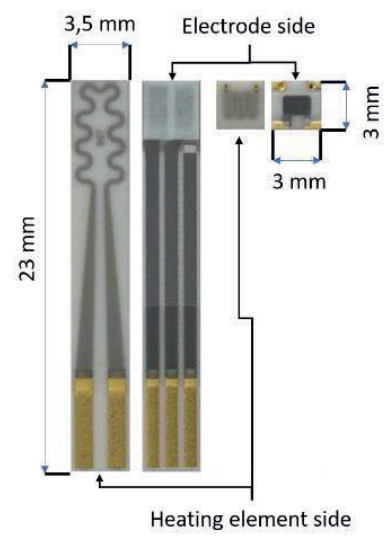

Figure 12: Picture comparing the fully fabricated LH 44 sensor in the standard size sensor and the miniaturized sensor.

A fully fabricated LH 44X3 was assembled in a Transistor Outline (TO) housing. A picture of the LH 44X3 in TO housing is shown in Figure 13.
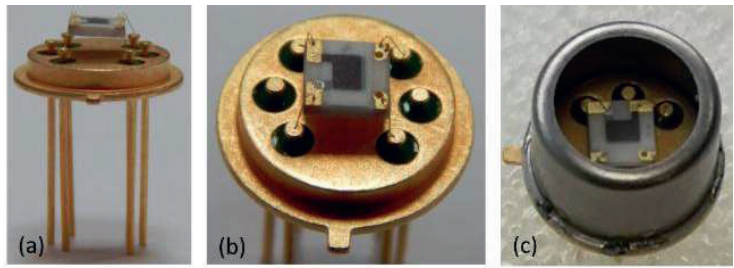

Figure 13: A fully fabricated $\mathrm{LH} 44 \mathrm{X} 3$ and assembled in a TO housing. (a) side view, (b) top view and (c) top view with full TO housing of the LH 44X3 sensors.

To achieve efficiency the new heater coil of LH 44X3 was designed thoroughly through elaborate scientific work.

The design simulation of the heating element was done within the framework of the project and it was optimized such that the minimum heating required to achieve the optimum sensor temperature was in the range of 0,8-1 W. The heating element was modelled in SolidWorks 2018 (Dessault Systémes). The Joule heating effects, voltage and temperatures were simulated and calculated using SolidWorks-Flow Simulation and Electronic Cooling modules for a current range from $0,55 \mathrm{~A}$ to $0,90 \mathrm{~A}$. A picture of the optimal heating element design obtained from the simulation is shown in Figure 14. 


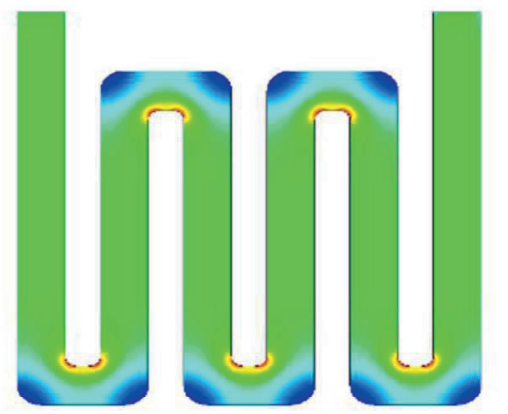

Figure 14: Picture of the optimal heating element design with Solidworks Flow Simulation module.

Figure 15, shows the calculated optimal operation point for $\mathrm{LH} 44 \mathrm{X} 3$ as required by specification at $0,7 \mathrm{~A}$ and $0,97 \mathrm{~V}$ with peak temperature of $454{ }^{\circ} \mathrm{C}$ in the centre reaching down to $440{ }^{\circ} \mathrm{C}$ at the outer edge of the $3 \mathrm{~mm} * 3 \mathrm{~mm}$ substrate resulting in a calculated power draw of $0,68 \mathrm{~W}$.

To verify the simulation a LH $44 \mathrm{X} 3$ sensor was fabricated and tested with the optimized heating element design. A picture of the fully fabricated heating element based on the obtained simulation results is shown in Figure 16.

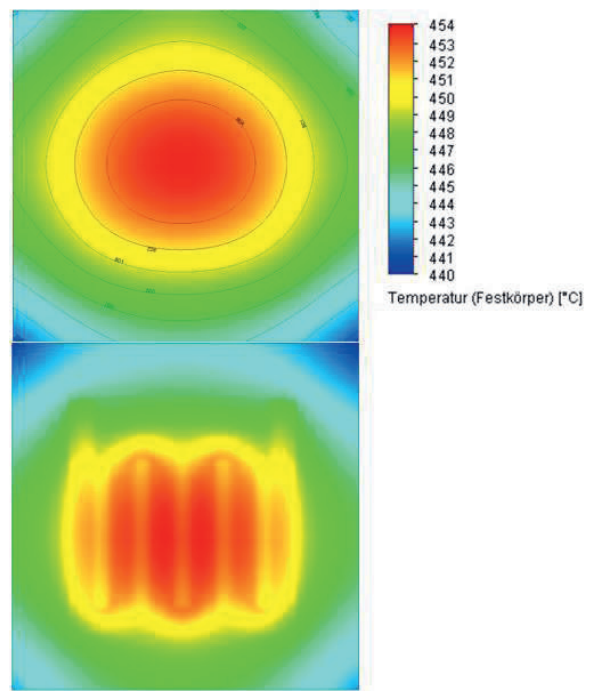

Figure 15: Simulation of the LH 44X3 sensor heating element, shown are resulting temperatures at the optimal working point of $0.7 \mathrm{~A}$.

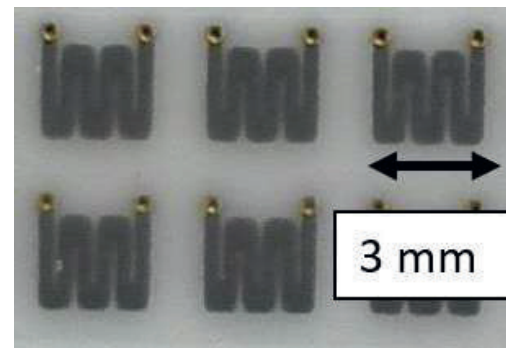

Figure 16: Optimized fabricated heating element of the LH 44 X3.

The initial testing of the LH 44X3 was successful with the optimized heating element. The fully fabricated and assembled LH 44X3 sensors were heated using a high precision power source, Keithley 2601B. The power source was set in current controlled mode of operation. The current was also measured using an Agilent 34401A digital multimeter. Experiments were conducted in order to achieve the sensor operating temperature from $400^{\circ} \mathrm{C}$ to $650^{\circ} \mathrm{C}$. The measured temperature was correlated with calculated power consumption values obtained from the simulation. Thermal imaging of the heated LH 44X3 sensor was performed using InfraTec VarioCam HD. A typical measured thermal image is presented in Figure 17. In the figure, the peak temperature in the middle of the sensor is about $450{ }^{\circ} \mathrm{C}$. The thermal image was done with an emissivity of 0,95 . The heating power was $1,18 \mathrm{~W}$. The difference between the simulated and the measured heating power is attributed to the thermal losses that occur due to the bonding wires and the TO-housing.

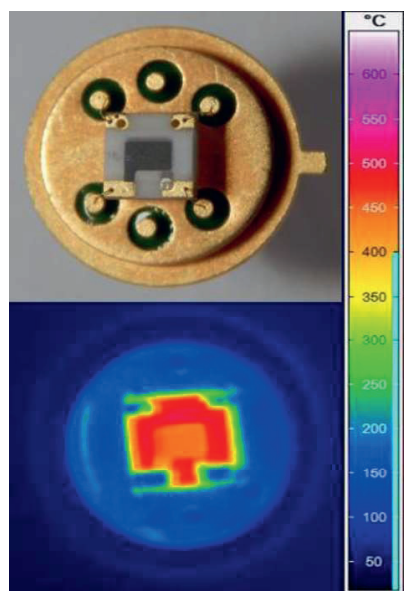

Figure 17: Thermal imaging of the LH 44X3 sensor with the optimized heating element. 


\section{Outlook}

The following aspects are currently work in progress:

- The further optimization of the heater power for the newly developed LH 44X3.

- The testing of the fully fabricated LH 44X3 with various hydrogen concentrations in air.

- The development of a simple electronic control unit for LH 44X3 is in progress.

Future work includes a system design for a mobile hydrogen leak detection unit that is driven by industrially available batteries or using a standard USB port.

\section{Acknowledgement}

We would like to thank the Karlsruhe Institute of Technology, PTKA-PFT and the Bundensministereim für Bildung und Foschung for the financial and administrative support for the project LHyCon (02P16K090).

\section{Reference}

[1] INFICON, "INFICON Holding AG," 29 04 2019. [Online]. Available: https://products.inficon.com/enus/navproducts/category/productgroup/leakdetectors/.

[2] M. Norio, S. Tomoaki, A. SriAyu, I. Hiroshi und Z. Serge, „A review of mixed-potential type zirconia-based gas sensors," lonics, Bd. 20, pp. 901925, 2014.

[3] M. Ralf, S. Kathy, F. Maximilian, G. Ulrich, B. Nicolae und W. Udo, „Solid State Gas Sensor Research in Germany - a Status Report," Sensors, Bd. 9, pp. 4323-4365, 2009.

[4] G. Fernando H., B. Eric L. und M. Rangachary, „THIN FILM MIXED POTENTIAL SENSORS“. USA Patent US7264700B1, 04092007.

[5] F. Hammer, „Vorrichtung und Verfahren zur Bestimmung der Konzentration eines Stoffes in einem Gas". Germany Patent DE102006054505A1, 29052008.

[6] LAMTEC, „LAMTEC Sensors and Systems for Combustion technology," [Online]. Available:

https://www.lamtec.de/en/products/se nsorik/carbosen-coe.html. [Zugriff am 2904 2019]. 